\title{
叶酸一磁性淀粉纳米颗粒的研制及其肿瘤靶向磁热疗 效应分析
}

郑元青, 童春义, 王贝, 谢英, 廖红东, 李丹 ${ }^{*}$, 刘选明 ${ }^{*}$

湖南大学生命科学与技术研究院, 生物能源与材料研究中心, 化学生物传感与计量学国家重点实验室, 长沙 410082

*联系人, E-mail: 1idanie@hotmail.com, sw xml@hnu.cn

2008-12-13 收稿, 2009-04-29 接受

湖南省农业生物纳米技术重大专项基金(编号: 03NKY1001)和国家高校“985 工程”创新平台项目资助

摘要 采用反相微乳液法制备了包裹磁流体的淀粉纳米颗粒 $\left(\mathrm{StNP} @ \mathrm{Fe}_{2} \mathrm{O}_{3}\right)$, 再经叶酸活性物 质(FA-PEG-NH2)修饰, 得到叶酸-磁性淀粉纳米颗粒(FA-StNP@ $\mathrm{Fe}_{2} \mathrm{O}_{3}$ ), 透射电子显微镜和激 光粒度分析仪检测显示, 所得颗粒的平均粒径约为 $250 \mathrm{~nm}$;邻菲罗啉法检测 FA-StNP@ $\mathrm{Fe}_{2} \mathrm{O}_{3}$ 的含铁量为 $2 \mathrm{mg} / \mathrm{g}$. FA-StNP@ $\mathrm{Fe}_{2} \mathrm{O}_{3}$ 纳米颗粒在交变磁场下作用 $30 \mathrm{~min}$, 可使环境温度 $\left(37^{\circ} \mathrm{C}\right)$ 升高到 $42 \sim 43^{\circ} \mathrm{C}$, 显示其具有一定的磁热效应. 将纳米颗粒分别与 HUEC-12 正常细胞和 Hela 肿瘤细胞共培养, 经交变磁场作用后, 通过四甲基偶氮唑盐比色法(MTT)实验、Hochest-PI 双

关键词 磁纳米颗粒 叶酸 肿瘤靶向性 磁热疗 细胞凋亡 染色分析、流式细胞仪技术检测了纳米颗粒在细胞水平的生物学效应, 结果表明, FA-StNP@ $\mathrm{Fe}_{2} \mathrm{O}_{3}$ 纳米颗粒在未加磁场时, 在一定浓度范围内对细胞的增殖无明显影响; 而在 一定交变磁场强度作用下则能有效地诱导 HeLa 细胞调亡, 细胞凋亡率为 $13.4 \%$, 普鲁士蓝染 色实验显示, 叶酸修饰有助于 FA-StNP $@ \mathrm{Fe}_{2} \mathrm{O}_{3}$ 纳米颗粒靶向识别 $\mathrm{HeLa}$ 细胞. 研究表明, FA-StNP@ $\mathrm{Fe}_{2} \mathrm{O}_{3}$ 纳米颗粒具有良好的肿瘤靶向磁热治疗效果, 可望应用于肿瘤的靶向治疗.

近年来，磁性纳米颗粒因其独特的性质被广泛 应用于生物治疗和诊断等领域. 其具有的表面化学 活性, 可以很容易地结合生物大分子, 通过在其表面 修饰上特异性的配体使之成为很好的靶向性载体 ${ }^{[1,2]}$. 磁性微粒在交变磁场作用下能吸收电磁波能量转化 为热能, 且可使热能局限于肿瘤组织, 当温度超过 $41^{\circ} \mathrm{C}$ 时可导致细胞的凋亡及坏死，因而可实现对肿 瘤的热疗 ${ }^{[3 \sim 5]}$.

在临床治疗中, 药物的低毒和高效一直是人们 研究的重点, 药物靶向传递的研究, 即如何发挥药物 高效治疗的效果越来越受到研究者的重视. 叶酸是 小分子量维生素, 相对于单分子抗体等蛋白质, 具有 结构稳定、价格低廉、无免疫原性等特点, 而且叶酸 与叶酸受体结合力强, 能被高效介导进入肿瘤细胞,
是一种很有应用价值的靶向物质 $[6]$. 根据叶酸受体 在绝大多数恶性肿瘤细胞膜内有大量表达而正常细 胞很少有表达这一特性, 人们将叶酸通过化学键连 接到药物载体以及它们制成的纳米颗粒上 ${ }^{[7 \sim 9]}$, 致使 叶酸/叶酸受体介导靶向传递成为该领域的研究热点 [10].

磁纳米颗粒研究发展至今, 颗粒的尺寸、表面分 子的修饰、靶向性等都成为人们所关注的重点 ${ }^{[11 ~ 13]}$. 为了进一步改善磁性纳米颗粒对细胞的亲和性和靶 向性, 提高热疗效果, 本文以天然多糖淀粉为原料, 通过反相微乳液法制备了磁性淀粉纳米颗粒; 然后 将结合有氨基的肿瘤细胞特异靶向分子叶酸对其表 面进行生物学修饰, 以体外培养的细胞为研究对象, 对制备的叶酸-磁性淀粉纳米颗粒(FA-StNP@ $\mathrm{Fe}_{2} \mathrm{O}_{3}$ ) 
的细胞内磁热疗诱导细胞调亡进行了研究. 实验结 果证实了磁性纳米颗粒表面经过靶向分子的修饰可 以达到对肿瘤细胞靶向识别和在细胞内富集的目的.

\section{1 材料与方法}

( i ) 仪器与试剂. 透射电子显微镜(JEOL-1230, 日本), 激光粒度分析仪(Zeta-sizer, Malvern, 英国), 高速离心机(5810 R Eppendorf, 德国), 高速低温离心 机(Himac CR 22G, Hitachi, 日本), 紫外分光光度计(北 京瑞利分析仪器厂), 菼光倒置显微镜 (Nikon $\mathrm{TE} 2000 \mathrm{u}$, 日本), $\mathrm{CO}_{2}$ 细胞培养箱(Thermo 3110, 美 国)，超纯水系统(Millipore，美国)，酶标仪(Bio-Rad， 美国), 交变磁场发射器 $(80 \mathrm{~Hz}, 10 \mathrm{kA} / \mathrm{m}$, 实验室自 制).

磁流体 $\gamma-\mathrm{Fe}_{2} \mathrm{O}_{3}$ 参照文献 [14]制备, FA-PEG-NH 按照文献 [6]合成, RPMI 1640 培养基粉剂购自美国 GIBCO公司, 小牛血清、青霉素和链霉素等均购自北 京鼎国生物技术公司, Hochest-PI染色剂购自美国 Invitrogen公司, 其他试剂均为国产分析纯. 人正常 细胞株HUEC-12(人脐静脉内皮细胞)、肿瘤细胞株 $\mathrm{HeLa}$ 均购自中南大学湘雅医学院细胞库, 由本实验 室进行常规培养.

(ii) 磁性淀粉纳米颗粒 $\left(\mathrm{StNP} @ \mathrm{Fe}_{2} \mathrm{O}_{3}\right)$ 的制备. 取 $0.1 \mathrm{~g} / \mathrm{mL}$ 淀粉溶液 $1 \mathrm{~mL}$, 沸水浴中加热至溶液澄 清, 冷却至室温. 甲苯和氯仿体积比 3:1 混合, 加入 占总体积 $2 \%$ 的表面活性剂 Span80, 搅拌混匀. 淀粉 液与磁流体混合, 加入到甲苯与氯仿的混合溶剂中 $\left(V_{\text {油 }} / V_{\text {水 }}=20 / 1\right)$, 搅拌至形成微小的乳液. 加入占淀粉 质量 $0.01 \%$ 的交联剂 $\mathrm{POCl}_{3}$, 继续搅拌反应 $1 \mathrm{~h}$, 用丙 酮和无水乙醇轮流洗涤乳液 3 次, 冷冻干燥, 得 $\mathrm{StNP} @ \mathrm{Fe}_{2} \mathrm{O}_{3}$.

(iii) $\mathrm{StNP} @ \mathrm{Fe}_{2} \mathrm{O}_{3}$ 的表面修饰. 参照文献 [10]对 $\mathrm{StNP} @ \mathrm{Fe}_{2} \mathrm{O}_{3}$ 进行叶酸分子的表面修饰. 首先取 $1 \mathrm{mg}$ 的 $\mathrm{St}-\mathrm{Fe}_{2} \mathrm{O}_{3}$ 完全分散于 $1 \mathrm{~mL} \mathrm{PBS}(\mathrm{pH}$ 7.0)中, 然后加 入适量的FA-PEG- $\mathrm{NH}_{2}$, 振荡反应 $6 \mathrm{~h}$, 冷冻干燥后, 得到淡黄色粉末, 即为FA-StNP@ $\mathrm{Fe}_{2} \mathrm{O}_{3}$ 颗粒.

(iv) FA-StNP@ $\mathrm{Fe}_{2} \mathrm{O}_{3}$ 的表征分析. 通过透射电 子显微镜和 Zeta 电位-粒度分析仪对磁性纳米颗粒的 大小、形貌以及粒度分布进行表征分析.

（v）铁含量分析. 取 $1 \mathrm{mg}$ 磁性纳米颗粒悬浮于 $1 \mathrm{~mL}$ 双蒸水中, 用 $\alpha$-淀粉酶消化除去淀粉, $37^{\circ} \mathrm{C}$ 振摇 $2 \mathrm{~h}$, 经稀释 100 倍后加入邻菲罗㕲染色液, 紫外可见
分光光度计测定 $510 \mathrm{~nm}$ 吸光度. 以分析纯 $\mathrm{Fe}_{2} \mathrm{O}_{3}$ 为标 准物质, 建立铁含量-吸光度标准曲线.

(vi) 磁热效应分析. 先将含磁性纳米颗粒的培 养液在 $37^{\circ} \mathrm{C}$ 恒温培养箱中孵育 $1 \mathrm{~h}$, 再在交变磁场下 处理不同时间, 用精密温度计测量溶液温度. 考察了 不同浓度磁纳米颗粒在磁场作用下的处理时间与环 境温度的变化情况.

(vii）四甲基偶氮唑盐比色法(MTT)实验. 选对 数生长期的细胞, 以 10000 个细胞/孔的密度接种于 细胞 96 孔板内, 每孔加入 $100 \mu \mathrm{L}$ 的完全培养基培养 $24 \mathrm{~h}$. 分别加入不同浓度梯度的 $\gamma-\mathrm{Fe}_{2} \mathrm{O}_{3}, \mathrm{StNP} @$ $\mathrm{Fe}_{2} \mathrm{O}_{3}$ 和 FA-StNP@ $\mathrm{Fe}_{2} \mathrm{O}_{3}$, 继续培养 $24 \mathrm{~h}$, 加入 $20 \mu \mathrm{L}$ MTT, 继续培养 $4 \mathrm{~h}$, 除去上层清液后, 用 DMSO 溶 解沉淀, 分光光度计测定 $570 \mathrm{~nm}$ 吸光度. 以空白处 理细胞组为对照组, 计算细胞抑制率和成活率.

(viii) Hochest-PI 双染色分析. 选对数生长期的细 胞, 以 10000 个细胞/孔的密度接种于细胞 6 孔板内, 加入 $2 \mathrm{~mL}$ 的完全培养基培养 $24 \mathrm{~h}$. 磁场处理(颗粒浓 度 $1 \mathrm{mmol} / \mathrm{L}$; 作用时间 $30 \mathrm{~min}$ ), 收集细胞后与 Hochest-PI 染色液混匀, 避光染色 $5 \mathrm{~min}$, 用培养基洗去 游离染色液, 重悬细胞, 细胞液滴在载玻片上, 用苂 光显微镜进行苂光染色观察. 以空白处理细胞组为 对照组.

（ix）流式细胞仪检测. 如 (viii) 节的方法处理细 胞后, 70\%冷乙醇固定过夜. 次日用 PBS 洗 2 次, 室 温下 PI 染色液(终浓度 $50 \mu \mathrm{g} / \mathrm{mL}$ ) 避光染色 $30 \mathrm{~min}$, 流式细胞仪检测细胞凋亡率. 以空白处理细胞组为 对照组.

(x) 普鲁士蓝染色分析. 用(viii)节的方法处理细 胞后, 分别加入等铁含量的 $\gamma-\mathrm{Fe}_{2} \mathrm{O}_{3}, \mathrm{StNP} @ \mathrm{Fe}_{2} \mathrm{O}_{3}$ 和 FA-StNP@ $\mathrm{Fe}_{2} \mathrm{O}_{3}$ 在培养血中, 并在磁场作用下分别 处理 $0.5 \mathrm{~h}$. 除去上层清液, PBS 洗两次, 加入普鲁士 蓝染液对处理细胞染色, PBS 洗两次, 除去多余的普 鲁士蓝染色液, 显微镜下观察. 以空白处理细胞组为 对照组.

\section{2 结果与讨论}

\subsection{FA-StNP@ $@ \mathrm{Fe}_{2} \mathrm{O}_{3}$ 的形貌特征分析}

图 1(A), (B), (C) 分别为磁流体 $\gamma-\mathrm{Fe}_{2} \mathrm{O}_{3}$ 以及 FA-StNP@ $\mathrm{Fe}_{2} \mathrm{O}_{3}$ 纳米颗粒的透射电子显微镜图和粒 径分布图. 由图可知, $\gamma-\mathrm{Fe}_{2} \mathrm{O}_{3}$ 被包裹到淀粉纳米颗粒结 构中, 颗粒呈核-壳结构, $\gamma-\mathrm{Fe}_{2} \mathrm{O}_{3}$ 的平均粒径约为 $6 \mathrm{~nm}$, 

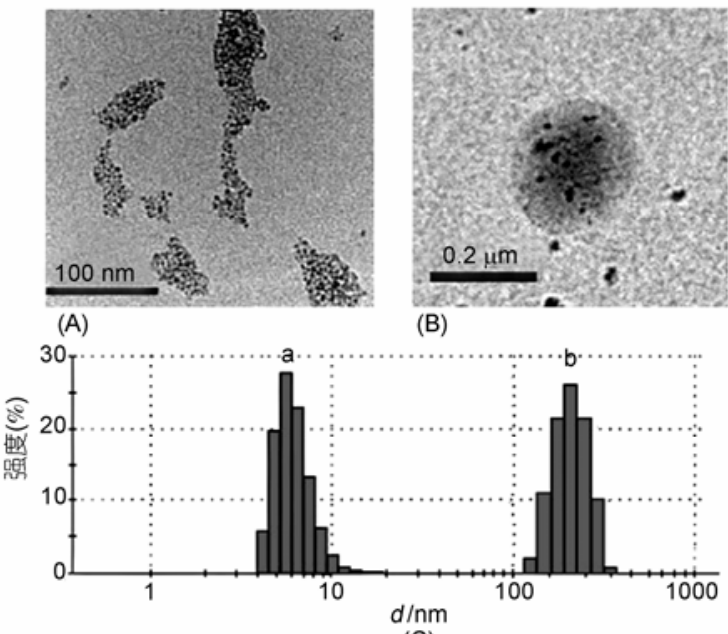

(C)

图 1 磁流体 $\gamma-\mathrm{Fe}_{2} \mathrm{O}_{3}$ 和 $\mathrm{FA}-\mathrm{StNP} @ \mathrm{Fe}_{2} \mathrm{O}_{3}$ 纳米颗粒的 表征图

(A)、(B) 磁流体 $\gamma-\mathrm{Fe}_{2} \mathrm{O}_{3}$ 和 $\mathrm{FA}-\mathrm{StNP} @ \mathrm{Fe}_{2} \mathrm{O}_{3}$ 纳米颗粒透射电 子显微镜图; (C) 纳米颗粒的粒径分布图, a: 磁流体 $\gamma-\mathrm{Fe}_{2} \mathrm{O}_{3}$; b: FA-StNP@ $\mathrm{Fe}_{2} \mathrm{O}_{3}$

分散系数为 $0.342, \mathrm{FA}-\mathrm{StNP} @ \mathrm{Fe}_{2} \mathrm{O}_{3}$ 粒径约为 $250 \mathrm{~nm}$, 分散系数为 0.422 . 结果表明, 制备的 FA-StNP@ $\mathrm{Fe}_{2} \mathrm{O}_{3}$ 形态规则, 分散性好.

\section{$2.2 \mathrm{FA}-\mathrm{StNP} @ \mathrm{Fe}_{2} \mathrm{O}_{3}$ 的铁含量分析}

邻菲罗啉法测定铁含量标准曲线为 $A=196.05 C+$ 0.0066, $R_{2}=0.9996$. 测定 FA-StNP $@ \mathrm{Fe}_{2} \mathrm{O}_{3}$ 纳米颗粒铁 含量约为 $2 \mathrm{mg} / \mathrm{g}, \gamma-\mathrm{Fe}_{2} \mathrm{O}_{3}$ 母液铁含量为 $306 \mathrm{mg} / \mathrm{g}$. 以 下实验中均以铁含量折算的摩尔浓度表示纳米颗粒 的使用浓度.

\section{$2.3 \mathrm{FA}-\mathrm{StNP} @ \mathrm{Fe}_{2} \mathrm{O}_{3}$ 对细胞增殖的影响}

磁热疗通常是建立在磁性纳米颗粒本身对细胞 无毒或低毒性的基础之上. 在未加磁场作用下，通过 MTT 实验考察纳米颗粒对细胞增殖的影响可反映纳 米颗粒的细胞毒性. 由图 2 可知, 在较低浓度下 $(0.5$, $1,5 \mathrm{mmol} / \mathrm{L})$, 纳米颗粒对共培养的 HeLa 细胞增殖的 影响不明显. 而在较高浓度下 $(10,20 \mathrm{mmol} / \mathrm{L})$, 不同 纳米颗粒的处理均使细胞的存活率有所下降. 说明纳 米颗粒对细胞增殖率的影响存在一定的浓度依赖性.

\subsection{FA-StNP@ $\mathrm{Fe}_{2} \mathrm{O}_{3}$ 磁热效应分析}

图 3 分析了不同处理时间下各种磁纳米颗粒的 磁热效应. 由图可知, 交流磁场作用 $30 \mathrm{~min}$, 含有磁 性纳米颗粒的培养基温度从 $37^{\circ} \mathrm{C}$ 上升到 $42 \sim 43^{\circ} \mathrm{C}$, 说 明磁性纳米颗粒均有明显的磁热效应. 其中磁流体

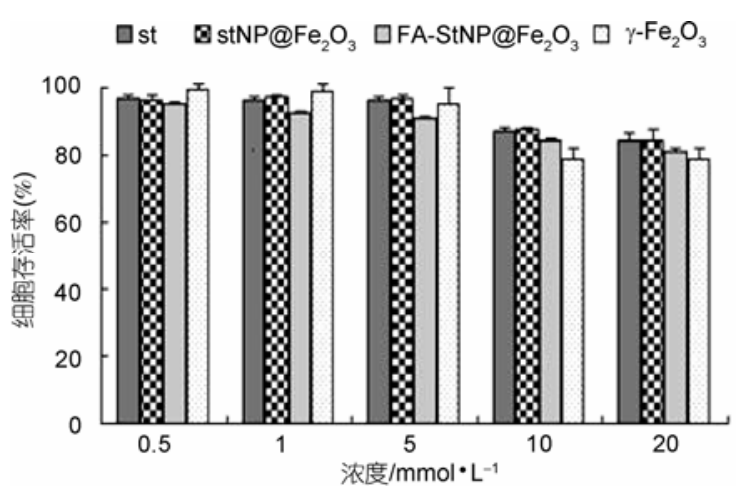

图 2 不同纳米颗粒作用下的细胞存活率

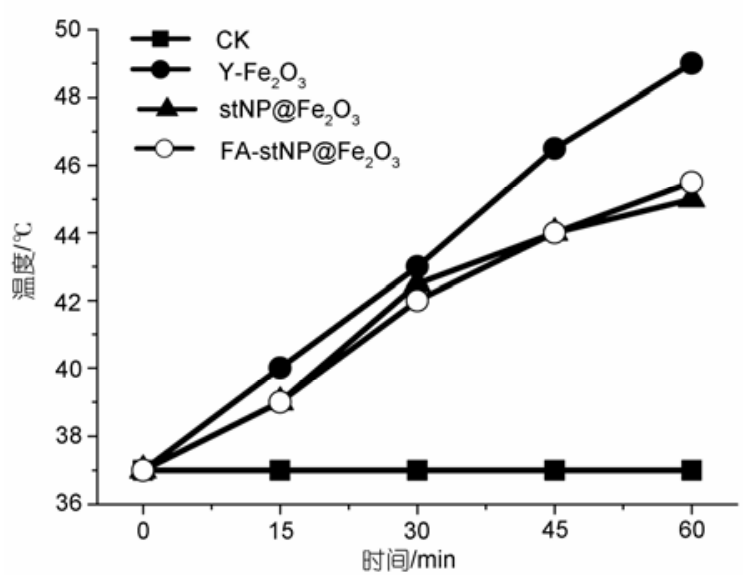

图 3 不同磁性纳米颗粒在交变磁场作用下的升温曲线图

的磁热效应要比包裹了磁流体的淀粉纳米颗粒强, 这可能是由于磁流体颗粒小, 分散性要比磁性淀粉纳 米颗粒好的缘故. 对于细胞生长来说, 温度达到 $42^{\circ} \mathrm{C}$, 热作用即能诱导细胞凋亡, 从而发挥热疗的效果 ${ }^{[15]}$.

基于 FA-StNP@ $\mathrm{Fe}_{2} \mathrm{O}_{3}$ 颗粒作用下的细胞成活率 实验和磁热效应分析的结果, 在下面的实验中, 选择 $1 \mathrm{mmol} / \mathrm{L}$ 的浓度、以交变磁场作用 $30 \mathrm{~min}$ 来考察磁 性纳米颗粒在细胞水平的生物学效应.

\section{5 细胞凋亡检测}

( i ) Hochest-PI 双染色实验. Hochest-PI 双染色 实验考察了不同纳米颗粒磁热疗诱导细胞调亡的情 况. 从图 4 可知, 在对肿瘤细胞 Hela 的处理实验中, 对照组细胞染色质均匀分布 (图 4(a),(e)), 而经 $\gamma-\mathrm{Fe}_{2} \mathrm{O}_{3}$ 和 FA-StNP@ $\mathrm{Fe}_{2} \mathrm{O}_{3}$ 磁性纳米颗粒处理后, 均 有部分细胞出现月牙状蓝色苂光, 在叠加视场中可 观察到染色质集中分布在细胞的一边, 呈明显的染 色质浓缩现象 (图 4(b) (d),(f) (h)), 说明不同的磁性 纳米颗粒在交变磁场作用下均可诱导肿瘤细胞凋亡. 
此外, 在图 4(b)和(f)中还观察到了红色苂光, 这提示 在交变磁场的作用下, 经 $\gamma-\mathrm{Fe}_{2} \mathrm{O}_{3}$ 颗粒处理的细胞除 发生了细胞调亡外, 还出现了部分细胞坏死. 图 5 是 不同纳米颗粒对人正常细胞 HUEC-12 的处理结果, 其中对照组细胞染色质均匀分布(图 5(a),(e)), 而经磁 性纳米颗粒处理后, 大多数 HUEC-12 细胞无明显凋 亡特征出现, 仅观察到极少数细胞有轻微的染色质 浓缩现象(图 5(b) (d),(f) (h)), 提示磁性纳米颗粒在 交变磁场作用下所导致的 HUEC-12 细胞凋亡现象没
有其在肿瘤细胞中的作用显著.

(ii) 流式细胞仪分析. 采用流式细胞术定量分 析了磁性纳米颗粒在交变磁场处理前后细胞凋亡水 平和调亡率的变化. 如图 6 所示, 不同磁性纳米颗粒 在没有交变磁场作用时，不会引起 HeLa 细胞凋亡， 其亚 G1 峰 (即调亡峰) 值均在 $1 \%$ 以内, 与对照组无 统计学上的差异. 而在交变磁场作用下, 磁性纳米颗 粒由于磁热效应可引起 HeLa 细胞凋亡, 均出现明显 的亚 G1 峰. 其中叶酸介导的磁性淀粉纳米颗粒 FA-

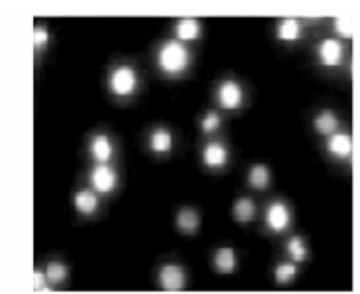

(a)

A组

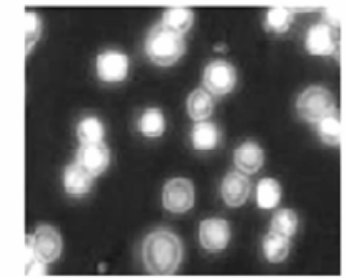

(e)

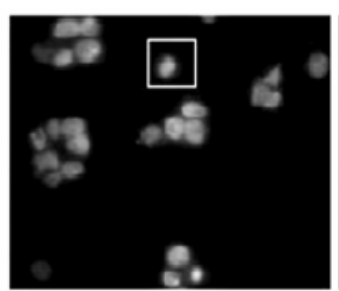

(b)

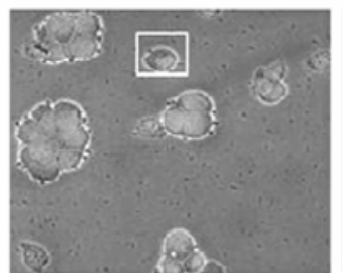

(f)

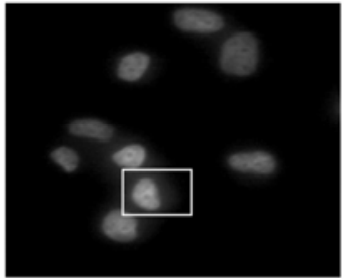

(c)

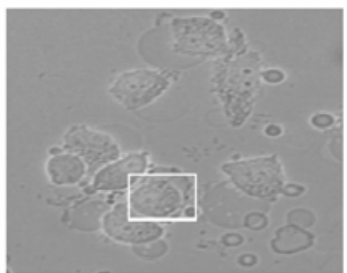

(g)

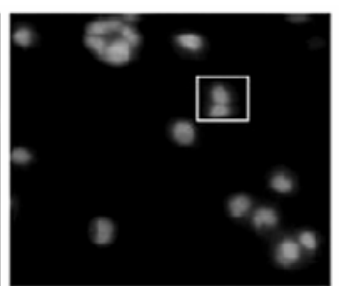

(d)

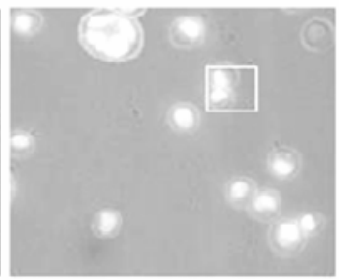

(h)

图 4 不同纳米颗粒磁场处理 HeLa 细胞后 Hochest-PI 染色结果 $(\times 200)$

$\mathrm{A}$ 组为苂光视场效果图, B 组为明视场和苂光视场叠加的效果图. (a),(e): 空白组; (b),(f): $\gamma-\mathrm{Fe}_{2} \mathrm{O}_{3}$ 组; (c), (g): $\mathrm{StNP}_{0} \mathrm{Fe}_{2} \mathrm{O}_{3}$ 组; (d),(h): FA-StNP@ $\mathrm{Fe}_{2} \mathrm{O}_{3}$ 组

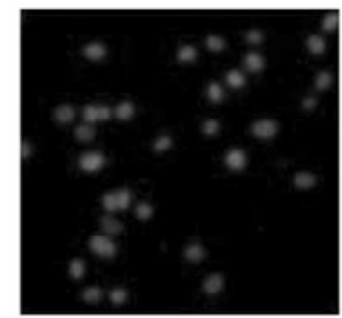

A组

(a)

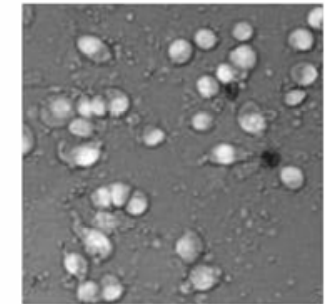

B组

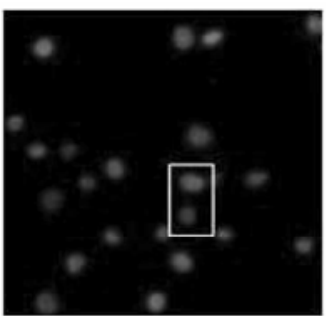

(b)

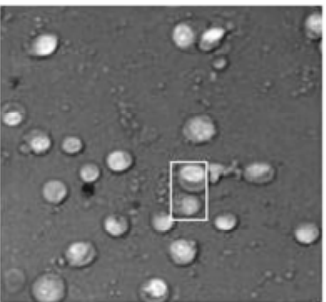

(f)

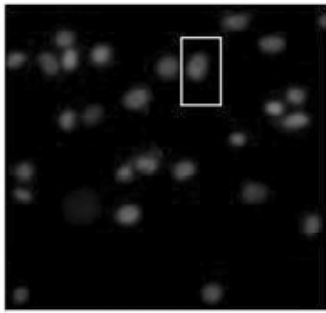

(c)

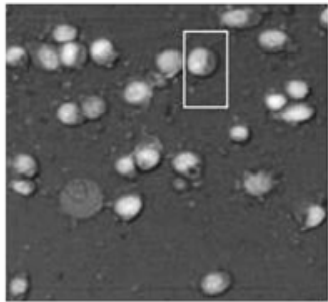

(g)

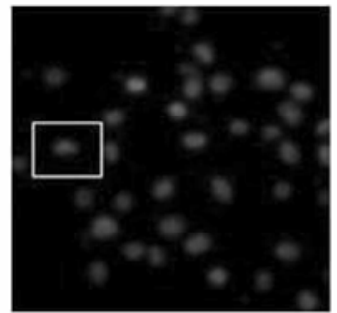

(d)

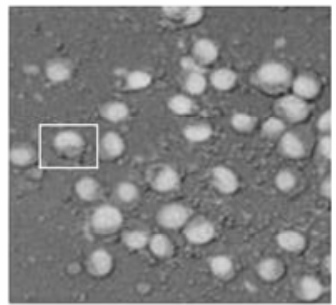

(h)

图 5 不同纳米颗粒磁场处理 HUEC-12 细胞后 Hochest-PI 染色结果 $(\times 200)$

$\mathrm{A}$ 组为荧光视场效果图, B 组为明视场和苂光视场叠加的效果图. (a),(e): 空白组; (b), (f): $\gamma-\mathrm{Fe}_{2} \mathrm{O}_{3}$ 组; (c), (g): $\mathrm{StNP} @ \mathrm{Fe}_{2} \mathrm{O}_{3}$ 组; (d), (h): FA-StNP@ $\mathrm{Fe}_{2} \mathrm{O}_{3}$ 组 


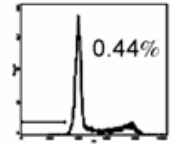

A对照组 (a)

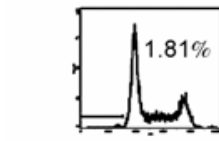

(e)

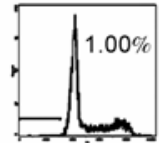

(b)

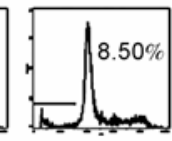

(f)

(g)

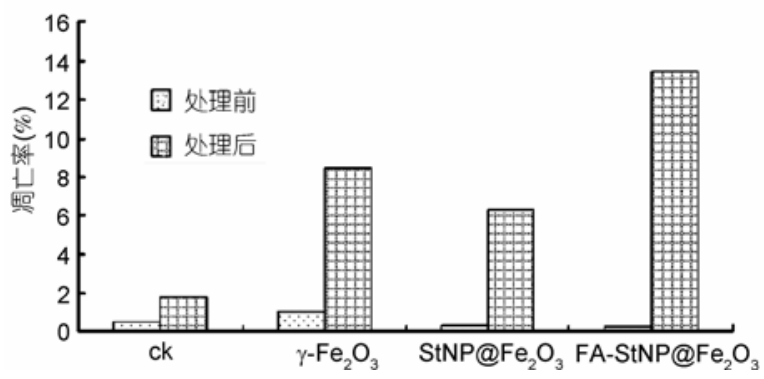

图 6 不同纳米颗粒经磁场处理前后对 HeLa 细胞 调亡的影响

(a) (d)：对照组 (即未处理组); (e) (h)：处理组; (a), (e)：空白组. (b), (f): $\gamma-\mathrm{Fe}_{2} \mathrm{O}_{3}$; (c),(g): StNP@ $\mathrm{Fe}_{2} \mathrm{O}_{3}$; (d),(h): FA-StNP@ $\mathrm{Fe}_{2} \mathrm{O}_{3}$

$\mathrm{StNP} @ \mathrm{Fe}_{2} \mathrm{O}_{3}$ 引起的调亡率为 $13.4 \%$, 高于没有叶酸介 导的 $\gamma-\mathrm{Fe}_{2} \mathrm{O}_{3}(8.5 \%)$ 和 $\mathrm{StNP} @ \mathrm{Fe}_{2} \mathrm{O}_{3}$ 磁性颗粒 $(6.3 \%)$. 结果表明, 通过叶酸修饰提高了磁性淀粉纳米颗粒 的肿瘤细胞靶向识别作用, 增加了细胞凋亡数量, 增 强了磁性纳米颗粒的磁热疗效果.

2.6 普鲁士蓝染色分析结果

为了进一步验证 FA-StNP $@ \mathrm{Fe}_{2} \mathrm{O}_{3}$ 纳米颗粒的靶
向性，用普鲁士蓝染色法分析了正常细胞和肿瘤细 胞吞噬纳米颗粒前后的细胞表面和吞噬进胞内的铁 含量. 如图 7 所示, 肿瘤细胞对照组没有加入磁铁颗 粒, 经普鲁士蓝染色后未出现任何着色, 而其他 3 个 加入磁性纳米颗粒的处理组均出现了明显的蓝色染 色. 比较 3 个磁性纳米组, 发现经叶酸修饰的纳米颗 粒 FA-StNP@ $\mathrm{Fe}_{2} \mathrm{O}_{3}$ 处理后的细胞染色程度要高于其 他两组, 证明了 FA-StNP@ $\mathrm{Fe}_{2} \mathrm{O}_{3}$ 的肿瘤细胞靶向性 使之能在细胞内有效地富集，在磁场作用下可诱导 较高的细胞调亡率. 而正常细胞由于很少表达叶酸 受体, 故只观察到较少的蓝色染色, 其中单纯磁流体 由于其粒径小, 较易被细胞吞噬, 但正常细胞对经淀 粉包裹之后的 $\mathrm{StNP} @ \mathrm{Fe}_{2} \mathrm{O}_{3}$ 的吞噬则大大减少. 正常 细胞对经叶酸修饰的淀粉纳米颗粒 FA-StNP@ $\mathrm{Fe}_{2} \mathrm{O}_{3}$ 的吞噬量也远远小于单纯磁流体的, 说明 FA-StNP@ $\mathrm{Fe}_{2} \mathrm{O}_{3}$ 的靶向热疗作用与其颗粒在细胞表 面的吸附和细胞吞噬量有直接关系, 提示该颗粒对 正常细胞的影响较小.

\section{3 结论}

近年来，肿瘤的细胞内热疗已成为肿瘤治疗 的一个热点. 热疗是通过加热肿瘤组织, 促进肿 瘤细胞凋亡的过程, 而热疗的加热源有多种, 磁 性材料是其中非常重要的一种. 本文成功制备了 FA$\mathrm{StNP} @ \mathrm{Fe}_{2} \mathrm{O}_{3}$ 纳米颗粒材料, 并通过一系列的生物学 效应实验, 发现磁性纳米颗粒经过表面叶酸修饰可

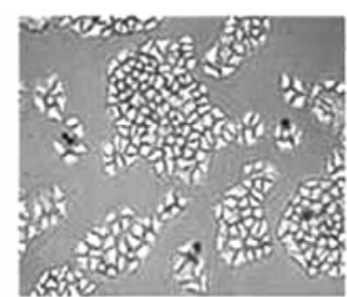

A组

(a)

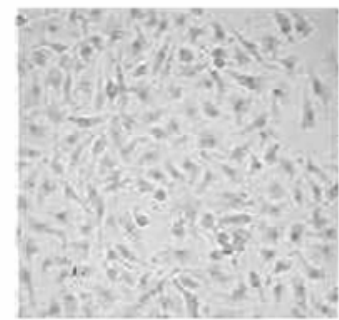

(e)

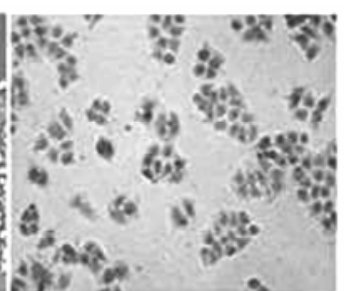

(b)

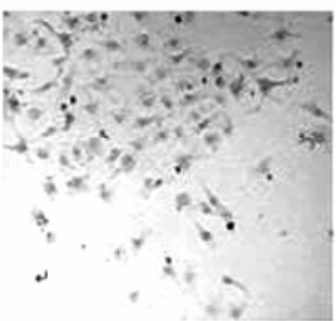

(f)

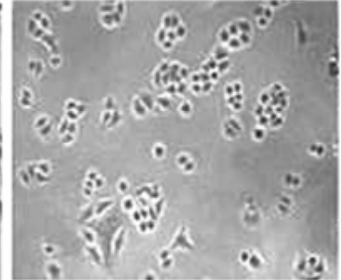

(c)

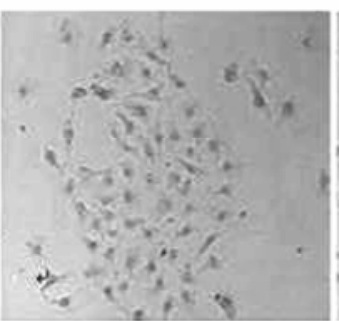

(g)

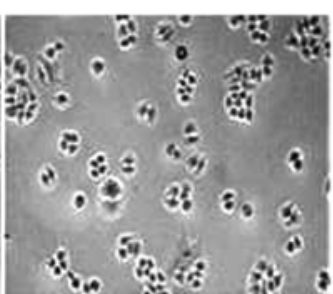

(d)

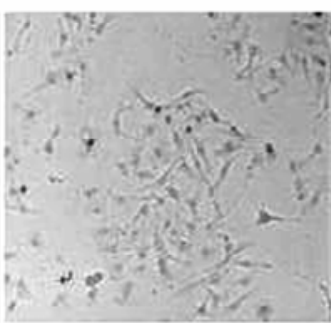

(h)

图 7 不同纳米颗粒进入细胞后的普鲁士蓝染色结果 $(\times 100)$

A 组: Hela 细胞组. (a) 对照组, (b) $\gamma-\mathrm{Fe}_{2} \mathrm{O}_{3}$ 组, (c) $\mathrm{StNP} @ \mathrm{Fe}_{2} \mathrm{O}_{3}$ 组, (d) FA-StNP@ $\mathrm{Fe}_{2} \mathrm{O}_{3}$ 组. B 组: HUCE-12 细胞组. (e) 对照组, (f) $\gamma-\mathrm{Fe}_{2} \mathrm{O}_{3}$ 组, (g) StNP@ $\mathrm{Fe}_{2} \mathrm{O}_{3}$ 组, (h) FA-StNP@ $\mathrm{Fe}_{2} \mathrm{O}_{3}$ 组 
以实现对肿瘤细胞的靶向识别和细胞内的富集. 表 明我们研制的 FA-StNP@ $\mathrm{Fe}_{2} \mathrm{O}_{3}$ 纳米颗粒具有良好
的肿瘤靶向磁热治疗效果，可望应用于肿瘤的靶向 治疗。

\section{致谢作者由衷感谢王海刚、林奕婷、薛昌刚对本实验的贡献.}

\section{参考文献}

1 Saini S, Sharma R, Baron R L, et al. Multicentre dose-ranging study on the efficacy of USPIO ferumoxtran-10 for liver MR imaging. Clin Radiol, 2000, 55: 690-695[doi]

2 Gupta A K, Gupta M. Synthesis and surface engineering of iron oxide nanoparticles for biomedical applications. Biomaterials, 2005, 26: $3995-4021 \underline{\text { [doi] }}$

3 马勇杰, 李红, 滒祝兵, 等. 细胞内磁热疗诱导人肺腺癌细胞 SPC-A1 调亡的体外实验研究. 生物医学工程学杂志, 2007, 24: 1305-1308

4 Xiao X X, He Q Q, Huang K L, et al. Possible magnetic multifunctional nanoplatforms in medicine. Med Hypotheses, 2007, 68: 680682 [doi]

5 Nanz D, Weishaupt D, Quick H H, et al. TE-switched double-contrast enhanced visualization of vascular system and instruments for MR-guided interventions. Magn Reson Med, 2000, 43: 645-648[doi]

6 肖苏尧，童春义，刘选明，等. 肿瘤靶向性药物载体叶酸-淀粉纳米颗粒的研制与应用. 科学通报, 2006, 51: 1151一1155

7 Liang B, He M L, Xiao Z P, et al. Synthesis and characterization of folate-PEG-grafted-hyperbranched-PEI for tumor-targeted gene delivery. Biochem Biophys Res Commun, 2008, 367: 874-880[ [doi]

8 Ana G, Carmen R L, Edison L S, et al. Microspheres containing lipid/chitosan nanoparticles complexes for pulmonary delivery of therapeutic proteins. Eur J Pharm Biopharm, 2008, 69: 83-93[doi]

张良珂, 侯世祥, 毛声俊, 等. 叶酸偶联白蛋白纳米粒肿瘤细胞靶向性研究. 四川大学学报(医学版), 2004, 35: 165-168

Sun H K, Ji H J, Cheol O J, et al. Folate receptor mediated intracellular protein delivery using PLL-PEG-FOL conjugate. J Control Release, 2005, 103: 625-634[doi]

11 Raynal I, Rrigent P, Peyramaure S, et al. Macrophage endocytosis of superparamagnetic iron oxide nanoparticles: mechanisms and comparison of ferumoxides and ferumoxtran-10. Invest Radiol, 2004, 39: 56-63 [doi]

12 Petri-Finka A, Chastellaina M, Juillerat-Jeanneretb F, et al. Development of functionalized superparamagnetic iron oxide nanoparticles for interaction with human cancer cells. Biomaterials, 2005, 26: 2685-2694[doi]

13 章林, 吴卫和, 王德平, 等. 纳米 MnZn 铁氧体微粒的磁热效应和细胞毒性. 中国生物医学工程学报, 2008, 27: 122一127

14 廖红东, 袁丽, 童春义, 等. 基于聚乙烯醇/ $\mathrm{Fe}_{2} \mathrm{O}_{3}$ 纳米颗粒的纤维素酶固定化. 高等学校化学学报, 2008, 29: 1564一-1568

15 Zhang Y, Zhang J. Surface modification of monodisperse magnetite nanoparticles for improved intracellular uptake to breast cancer cells. J Colloid Interface Sci, 2005, 283: 352-357 $\underline{\text { [doi] }}$ 